\title{
Survey on Aesthetic Vulvovaginal Procedures: What do Portuguese Doctors and Medical Students Think?
}

\section{Inquérito sobre procedimentos estéticos vulvovaginais: qual a opinião dos médicos e estudantes de medicina portugueses?} \author{
Fernanda Águas ${ }^{4}$

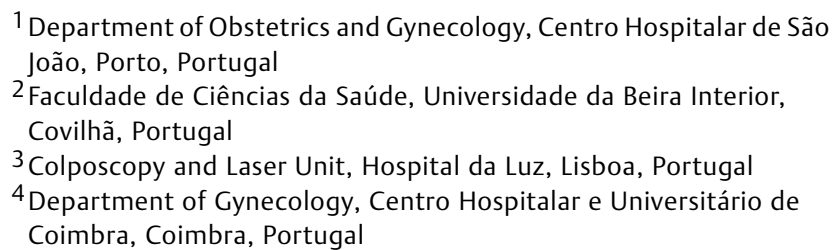

Pedro Vieira-Baptista ${ }^{1}$ Joana Lima-Silva ${ }^{1}$ José Fonseca-Moutinho ${ }^{2}$ Virgínia Monteiro ${ }^{3}$

Address for correspondence Pedro Vieira Baptista, MD, Departmento de Obstetrícia e Ginecologia, Centro Hospitalar de São João, Alameda Prof. Hernâni Monteiro, 4200-319, Porto, Portugal (e-mail: pedrovieirabaptista@gmail.com).

Rev Bras Ginecol Obstet 2017;39:415-423.

Objective To assess the medical doctors and medical students' opinion regarding the evidence and ethical background of the performance of vulvovaginal aesthetic procedures (VVAPs).

Methods Cross-sectional online survey among 664 Portuguese medical doctors and students.

Results Most participants considered that there is never or there rarely is a medical reason to perform: vulvar whitening (85.9\% [502/584]); hymenoplasty (72.0\% [437/607]); mons pubis liposuction (71.6\% [426/595]); "G-spot" augmentation (71.0\% [409/576]); labia majora augmentation (66.3\% [390/588]); labia minora augmentation (58.3\% [326/559]); or laser vaginal tightening (52.3\% [313/599]). Gynecologists and specialists were more likely to consider that there are no medical reasons to perform VVAPs; the opposite was true for plastic surgeons and students/residents.

Hymenoplasty raised ethical doubts in $51.1 \%$ (283/554) of the participants. Plastic surgeons and students/residents were less likely to raise ethical objections, while the opposite was true for gynecologists and specialists.

Most considered that VVAPs could contribute to an improvement in self-esteem ( $92.3 \%$ [613/664]); sexual function (78.5\% [521/664]); vaginal atrophy (69.9\% [464/664]); quality of life (66.3\% [440/664]); and sexual pain (61.4\% [408/664]).

Conclusions While medical doctors and students acknowledge the lack of evidence and scientific support for the performance of VVAPs, most do not raise ethical objections about them, especially if they are students or plastic surgeons, or if they have had or have considered having plastic surgery. received January 4, 2017 accepted April 17, 2017 published online June 23, 2017
DOI https://doi.org/ 10.1055/s-0037-1603967. ISSN 0100-7203.
Copyright $\odot 2017$ by Thieme Revinter

Publicações Ltda, Rio de Janeiro, Brazil
License terms

(ㄷ) (i) $\ominus$ (5) 


\section{Resumo}
Palavras Chave
- ninfoplastia
- himenoplastia
- cirurgia estética
- ética
- cirurgia vulvar
- laser

Objetivos Avaliar a opinião de médicos e estudantes de medicina relativamente à evidência e contexto ético para a realização de procedimentos estéticos vulvovaginais (PEVVs).

Métodos Estudo transversal, consistindo de inquérito online a 664 médicos e estudantes de medicina portugueses.

Resultados A maioria dos participantes considerou que nunca ou raramente há uma razão médica para a realização de: branqueamento vulvar (85,9\% [502/584]); himenoplastia (72,0\% [437/607]); lipoaspiração do mons pubis (71,6\% [426/595]); aumento do "ponto G" (71,0\% [409/576]); aumento dos grandes lábios (66,3\% [390/588]); aumento dos pequenos lábios (58,3\% [326/559]) ou aperto vaginal com laser (52,3\% [313/599]). Ser ginecologista e especialista associou-se a maior probabilidade de considerar não haver razões médicas para a realização de PEVV; o oposto foi verdade para os cirurgiões plásticos e estudantes/internos.

A himenoplastia levantou dúvidas em termos éticos em 51,1\% (283/554) dos participantes. Cirurgiões plásticos e estudantes/internos relataram menos dúvidas em termos éticos; o oposto foi verdade para os ginecologistas ou especialistas.

A maioria considerou que os $\mathrm{PEVV}$ s podem contribuir para uma melhoria na autoestima (92,3\% [613/664]); função sexual (78,5\% [521/664]); atrofia vaginal (69,9\% [464/664]); qualidade de vida (66,3\% [440/664]); e dor sexual (61,4\% [408/664]).

Conclusões Ainda que os médicos e estudantes de medicina reconheçam a falta de evidência e bases científicas para a realização de PEVVs, a maioria não levanta objecções em termos éticos, especialmente se forem estudantes, cirurgiões plásticos, ou se eles próprios tiverem sido submetidos a cirurgia plástica ou considerem vir a sê-lo.

\section{Introduction}

Vulvovaginal aesthetic procedures (VVAPs), also referred to as "intimate surgery" or "female cosmetic genital surgery", are a trending topic in the media that has been poorly addressed in the medical literature so far. Despite being available all over the world, these procedures are a marginal, gray area of medicine: there is no coherent classification of the procedures, and there is an evident lack of good quality, long-term, randomized, independent studies. ${ }^{1}$ This lack of evidence has not restricted the performance or advertisement of these procedures, which is easier than ever before in this digital era, due to the inability of other health professionals and/or medical societies to filter the information available. ${ }^{2}$ It has been shown that these advertisements are mostly inaccurate, omit risks and complications, and exaggerate the potential benefits. ${ }^{2,3}$ There is also indirect pressure to perform these procedures, since the pornographic industry and the media in general have created a new standard for the ideal "normal" vulva: ${ }^{4}$ hairless and with the labia minora totally hidden by the labia majora; anything that is different from this pre-pubertal model is now considered unaesthetic, abnormal and, thus, eligible for correction or perfection by the surgeon's blade or laser.

Medical societies are slowly starting to take public positions and publish guidelines, ${ }^{5-8}$ and more of them are expected to do the same in the near future. In the absence of good scientific evidence, it is difficult to assure the fulfillment of all medical ethical principles, which these guidelines must encompass: although the autonomy principle is the most important, in this setting, the principle of non-maleficence must not be disregarded.

Given the availability of VVAPs, the number of women demanding it, and the lack of data, it is critically important to know the clinicians' perception, knowledge and personal opinion on this topic. This could help in the development of reasonable and fair guidelines, while providing information for the public in general.

This study aimed at evaluating the opinions of medical doctors and medical students on the existence of any medical justification or ethical concerns regarding the different available VVAPs, as well as which factors might affect their opinion.

\section{Methods}

A cross sectional study was performed between September 01, 2015 and February 28, 2016. The study was approved by the Ethical Committee of one of our institutions. The participants were informed of the objectives of the study, and total confidentiality of the data was assured. Consent for participation was declared prior to the questionnaire. The eligibility criteria were: being a medical student at a Portuguese University, or being a medical doctor working in Portuguese territory.

Due to the unavailability of validated scales, a web-based (https://docs.google.com/forms/, Mountain View, CA, USA) questionnaire was specifically designed by the authors, following the checklist for reporting results of internet surveys (CHERRIES) 
protocol. ${ }^{9}$ It consisted of 25 questions, divided into 3 sections. The first section was intended to characterize the demographics of the participants (age, district of residence), differentiation (student, resident or specialist), specialty, type of practice (public and/or private), and if the participants themselves have had or have considered having plastic surgery (yes/no).

In the second section, the participants' opinion about any medical justification for, and ethical objections against, VVAPs was addressed. Given that there is currently no coherent nomenclature for these procedures, they were explained and clarified whenever necessary.

In this section of the questionnaire, the 3 or 4-point Likert scales were used. The participants were asked to answer whether several VVAPs were medically justifiable, with the following answer options: "it is never justifiable," "it is rarely justifiable," "it is sometimes justifiable," or "it is frequently justifiable"). For the purpose of analysis, the first two options were considered as a single answer, and the same was done with the last two.

For the question of whether or not a procedure was ethical, considering the scientific data available, the answer options included: "clearly against medical ethics," "doubtful in terms of medical ethics," and "no medical ethical objections." For the purpose of analysis, the first two options were grouped and compared against "no medical ethical objections."

In the third section, the participants were questioned about the possible benefits of VVAPs, and general statements and guidance on this topic were evaluated. A set of statements concerning practical issues regarding VVAPs (minimum age of performance, whether or not it should be performed in public hospitals, etc.) was evaluated using a 4-point Likert scale with the following options: "totally agree," "partially agree," "neither agree nor disagree," and "totally disagree."

Several potential benefits were presented, and the participants had to choose whether they agreed or not with the statements (yes/no).

There was an option of "don't answer/don't know" in the second and third sections of the questionnaire, but not on the first one.

The online survey was sent to the target population via email (collected from the databases of medical societies), and posted in the Web sites of medical societies and in specific social network groups (exclusively for doctors/medical students). According to the number of sent emails and the number of members on the social network groups, it was estimated that the questionnaire reached around 4,000 potential participants. According to the estimated size of the population of doctors and medical students, a sample of 382 would be enough to achieve a confidence level of $95 \%$ and a confidence interval of $5 \%$ (http://www.surveysystem.com/sscalc.htm, Sebastopol, CA, USA).

The statistical analysis was performed using the Microsoft Excel 2011 software (Microsoft Corporation, Redmond, WA, USA) and the Statistical Package for the Social Sciences (SPSS) software, version 20.0 (IBM Corp., Armonk, NY, USA). The chisquared test was used for the nominal variables, and the Student's $t$-test for the continuous variables. A $p<0.05$ was considered statistically significant.

\section{Results}

Questionnaires from 664 participants were obtained. The largest group of respondents were specialists (37.0\% [246/664], followed by students (34.2\% [227/664]) and residents (28.8\% [191/664]). The age of the sample ranged from 18 to 69 years $(32.9 \pm 12.15$ years old), and most participants were female (67.2\% [442/664]).

About one third had had or had considered having plastic surgery (29.7\% [188/633]), and there were no differences regarding the stage of medical differentiation (specialists - $27.9 \%$ [63/226] versus residents - 30.1\% [57/189] versus students $31.2 \%$ [68/218]; $p=0.88$ ). However, women were more likely to consider plastic surgery for themselves (34.1\% [145/425] versus 20.7\% [43/208]). Additionally, plastic surgeons (residents or specialists) or students intending to be become plastic surgeons were nearly twice as likely to report this than gynecologists (50.0\% [13/26] versus 26.7\% [36/135], $p=0.018]$ ).

Most specialists work in private practice (exclusively or not) $(65.9 \%$ [162/246]).

The representation by specialties (students were divided according to the specialty they intend to choose) was: gynecology (25.4\%; 155/611); plastic surgery (4.2\%; 28/611); other surgical specialty (14.2\%; 94/611); and other non-surgical specialty (50.3\%; 334/611).

More than half of the participants considered that there never or there rarely exists a medical reason to perform the following: vulvar whitening (85.9\% [502/584]); hymenoplasty (72.0\% [437/607]); liposuction of the mons pubis (71.6\% [426/595]); “G-spot” augmentation (71.0\% [409/576]); augmentation of the labia majora (66.3\% [390/588]); augmentation of the labia minora (58.3\% [326/559]); and vaginal tightening with laser (52.3\% [313/599]). Laser treatment of vaginal atrophy and nymphoplasty were the procedures that most participants considered as having a medical indication (77.2\% [467/605] and 74.7\% [430/576] respectively) (-Fig. 1).

The answers in this topic were independent of the fact that the respondents worked in private practice or not, of if they had had or had considered having plastic surgery themselves. Gender did not influence this opinion, except in the case of liposuction of the mons pubis (female - 74.1\% [303/409] versus male - 66.1\% [123/186]; $p=0.046)$ and vulvar whitening (female - 89.1\% [351/394] versus male $79.5 \%$ [151/190]; $p=0.002$ ). Gynecologists were much more likely to consider that there is no medical reason to perform these procedures, while the opposite was true for plastic surgeons. The answers of the group of other medical specialties, which was considered as a whole, scored inbetween. The grade of differentiation also had an influence on the outcome, with specialists more likely to consider there was an absence of medical justification for the procedures, while students were more likely to consider the opposite (-Table 1).

Only hymenoplasty raised ethical doubts in more than half of the participants (51.1\% [283/554]). A quarter or less raised concerns about reduction of the labia majora $(25.2 \%$ [138/547]), laser for the treatment of vaginal atrophy (20.7\% [112/541]), and nymphoplasty (18.5\% [95/514]) (-Fig. 2). 
418 Survey on Aesthetic Vulvovaginal Procedures Vieira-Baptista et al.

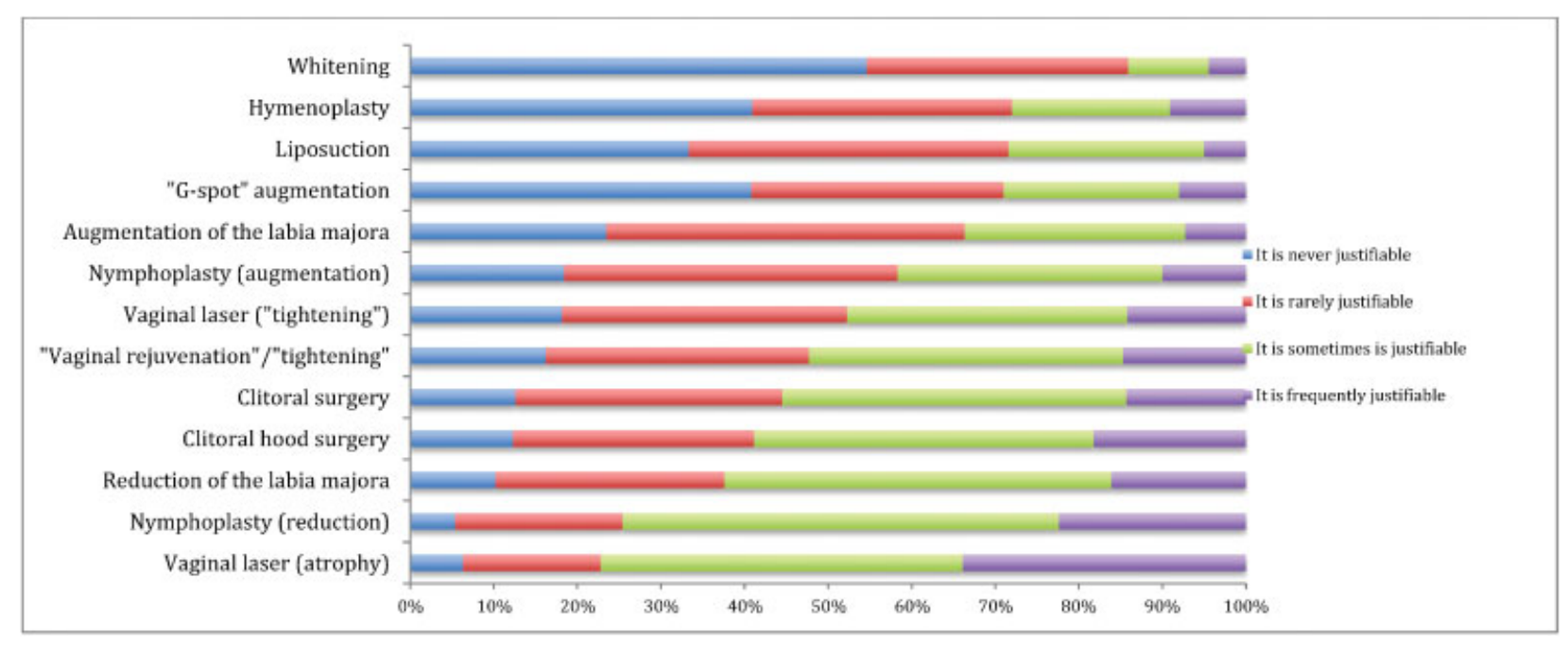

Fig. 1 Participants' opinion in terms of medical justification for the performance of VVAPs.

The gender of the participants and the fact that they worked in private practice did not influence the opinion about the ethical issues associated with these procedures. On the contrary, specialty had an effect, since being a plastic surgeon was associated with a lower likelihood of having ethical objections, while being a gynecologist was associated with the opposite. Those who had considered having or who had had plastic surgery themselves were less likely to raise ethical concerns; the same was true for the residents, and even more so for the students (-Table 2 ).

Only $2.1 \%$ (14/664) of the participants considered that there is never any benefit from VVAPs. Most participants considered that they could contribute to an improvement in: self-esteem (92.3\% [613/664]); sexual function $(78.5 \%$

Table 1 Percentage of participants who answered that a specific procedure did not or rarely had a medical indication

\begin{tabular}{|c|c|c|c|c|c|c|c|c|c|c|c|}
\hline & \multicolumn{3}{|l|}{$\begin{array}{l}\text { Gender } \\
\%(n / N)\end{array}$} & \multicolumn{3}{|c|}{$\begin{array}{l}\text { Private practice* } \\
\%(n / N)\end{array}$} & \multicolumn{5}{|l|}{$\begin{array}{l}\text { Specialty } \\
\%(n / N)\end{array}$} \\
\hline & Female & Male & $p$ & Yes & No & $p$ & ObGyn & $\begin{array}{l}\text { Plastic } \\
\text { surgery }\end{array}$ & $\begin{array}{l}\text { Other } \\
\text { non-surgical }\end{array}$ & $\begin{array}{l}\text { Other } \\
\text { surgical }\end{array}$ & $p$ \\
\hline $\begin{array}{l}\text { Vaginal laser } \\
\text { (atrophy) }\end{array}$ & $\begin{array}{l}23.2 \% \\
(96 / 413)\end{array}$ & $\begin{array}{l}21.9 \% \\
(42 / 192)\end{array}$ & 0.709 & $\begin{array}{l}27.0 \% \\
(38 / 141)\end{array}$ & $\begin{array}{l}30.0 \% \\
(23 / 76)\end{array}$ & 0.873 & $\begin{array}{l}33.8 \% \\
(50 / 148)\end{array}$ & $\begin{array}{l}4.2 \% \\
(1 / 24)\end{array}$ & $\begin{array}{l}21.7 \% \\
(65 / 300)\end{array}$ & $\begin{array}{l}17.6 \% \\
(15 / 85)\end{array}$ & 0.001 \\
\hline $\begin{array}{l}\text { Nymphoplasty } \\
\text { (reduction) }\end{array}$ & $\begin{array}{l}25.3 \% \\
(47 / 184)\end{array}$ & $\begin{array}{l}25.3 \% \\
(99 / 392)\end{array}$ & 0.941 & $\begin{array}{l}32.6 \% \\
(44 / 135)\end{array}$ & $\begin{array}{l}31.5 \% \\
(23 / 73)\end{array}$ & 1.000 & $\begin{array}{l}30.9 \% \\
(47 / 152)\end{array}$ & $\begin{array}{l}0.0 \% \\
(0 / 26)\end{array}$ & $\begin{array}{l}24.5 \% \\
(68 / 278)\end{array}$ & $\begin{array}{l}26.2 \% \\
(21 / 80)\end{array}$ & 0.009 \\
\hline $\begin{array}{l}\text { Reduction of the } \\
\text { labia majora }\end{array}$ & $\begin{array}{l}38.8 \% \\
(161 / 415)\end{array}$ & $\begin{array}{l}35.1 \% \\
(68 / 194)\end{array}$ & 0.374 & $\begin{array}{l}52.7 \% \\
(78 / 148)\end{array}$ & $\begin{array}{l}48,1 \% \\
(37 / 77)\end{array}$ & 0.508 & $\begin{array}{l}59.2 \% \\
(90 / 152)\end{array}$ & $\begin{array}{l}16.7 \% \\
(4 / 24)\end{array}$ & $\begin{array}{l}31.2 \% \\
(95 / 304)\end{array}$ & $\begin{array}{l}36.5 \% \\
(31 / 85)\end{array}$ & $<0.001$ \\
\hline $\begin{array}{l}\text { Clitoral hood } \\
\text { surgery }\end{array}$ & $\begin{array}{l}43.1 \% \\
(172 / 399)\end{array}$ & $\begin{array}{l}37.1 \% \\
(72 / 194)\end{array}$ & 0.164 & $\begin{array}{l}53.4 \% \\
(79 / 148)\end{array}$ & $\begin{array}{l}54.1 \% \\
(40 / 74)\end{array}$ & 0.924 & $\begin{array}{l}61.7 \% \\
(92 / 149)\end{array}$ & $\begin{array}{l}20,0 \% \\
(5 / 25)\end{array}$ & $\begin{array}{l}35.6 \% \\
(104 / 292)\end{array}$ & $\begin{array}{l}38.6 \% \\
(32 / 83)\end{array}$ & $<0.001$ \\
\hline Clitoral surgery & $\begin{array}{l}44.1 \% \\
(173 / 392)\end{array}$ & $\begin{array}{l}45.2 \% \\
(85 / 182)\end{array}$ & 0.806 & $\begin{array}{l}59.9 \% \\
(88 / 147)\end{array}$ & $\begin{array}{l}62.0 \% \\
(44 / 71)\end{array}$ & 0.765 & $\begin{array}{l}65.8 \% \\
(96 / 146)\end{array}$ & $\begin{array}{l}25 \% \\
(6 / 24)\end{array}$ & $\begin{array}{l}38.1 \% \\
(107 / 281)\end{array}$ & $\begin{array}{l}41.2 \% \\
(35 / 85)\end{array}$ & $<0.001$ \\
\hline $\begin{array}{l}\text { Vaginal } \\
\text { rejuvenation }\end{array}$ & $\begin{array}{l}50.0 \% \\
(208 / 416)\end{array}$ & $\begin{array}{l}42.9 \% \\
(84 / 196)\end{array}$ & 0.099 & $\begin{array}{l}42.3 \% \\
(63 / 149)\end{array}$ & $\begin{array}{l}48.1 \% \\
(37 / 77)\end{array}$ & 0.408 & $\begin{array}{l}55.8 \% \\
(86 / 154)\end{array}$ & $\begin{array}{l}30.8 \% \\
(8 / 26)\end{array}$ & $\begin{array}{l}45.2 \% \\
(137 / 303)\end{array}$ & $\begin{array}{l}44.6 \% \\
(37 / 83)\end{array}$ & 0.043 \\
\hline Laser (tightening) & $\begin{array}{l}54.6 \% \\
(224 / 410)\end{array}$ & $\begin{array}{l}47.1 \% \\
(89 / 189)\end{array}$ & 0.086 & $\begin{array}{l}54.0 \% \\
(75 / 139)\end{array}$ & $\begin{array}{l}54.5 \% \\
(42 / 77)\end{array}$ & 0.934 & $\begin{array}{l}63.3 \% \\
(93 / 147)\end{array}$ & $\begin{array}{l}40.0 \% \\
(10 / 25)\end{array}$ & $\begin{array}{l}48.0 \% \\
(144 / 300)\end{array}$ & $\begin{array}{l}50.0 \% \\
(40 / 80)\end{array}$ & 0.012 \\
\hline $\begin{array}{l}\text { Nymphoplasty } \\
\text { (augmentation) }\end{array}$ & $\begin{array}{l}59.3 \% \\
(224 / 378)\end{array}$ & $\begin{array}{l}56.4 \% \\
(102 / 181)\end{array}$ & 0.514 & $\begin{array}{l}66.4 \% \\
(89 / 134)\end{array}$ & $\begin{array}{l}67.6 \% \\
(48 / 71)\end{array}$ & 0.864 & $\begin{array}{l}74.7 \% \\
(109 / 146)\end{array}$ & $\begin{array}{l}56.0 \% \\
(14 / 25)\end{array}$ & $\begin{array}{l}55.0 \% \\
(149 / 271)\end{array}$ & $\begin{array}{l}46.2 \% \\
(36 / 78)\end{array}$ & $<0.001$ \\
\hline $\begin{array}{l}\text { Augmentation of } \\
\text { the labia majora }\end{array}$ & $\begin{array}{l}65.7 \% \\
(262 / 399)\end{array}$ & $\begin{array}{l}67.7 \% \\
(128 / 189)\end{array}$ & 0.621 & $\begin{array}{l}70.3 \% \\
(102 / 145)\end{array}$ & $\begin{array}{l}73.3 \% \\
(55 / 75)\end{array}$ & 0.642 & $\begin{array}{l}78.9 \% \\
(116 / 147))\end{array}$ & $\begin{array}{l}60.9 \% \\
(14 / 23)\end{array}$ & $\begin{array}{l}62.3 \% \\
(182 / 292)\end{array}$ & $\begin{array}{l}65.1 \% \\
(54 / 83)\end{array}$ & 0.005 \\
\hline $\begin{array}{l}\text { "G-spot" } \\
\text { augmentation }\end{array}$ & $\begin{array}{l}71.8 \% \\
(285 / 397)\end{array}$ & $\begin{array}{l}69.3 \% \\
(124 / 179)\end{array}$ & 0.538 & $\begin{array}{l}84.2 \% \\
(112 / 133)\end{array}$ & $\begin{array}{l}77.1 \% \\
(54 / 70)\end{array}$ & 0.215 & $\begin{array}{l}86.4 \% \\
(121 / 140)\end{array}$ & $\begin{array}{l}56.0 \% \\
(14 / 25)\end{array}$ & $\begin{array}{l}69.0 \% \\
(196 / 284)\end{array}$ & $\begin{array}{l}61.4 \% \\
(51 / 83)\end{array}$ & $<0.001$ \\
\hline Liposuction & $\begin{array}{l}74.1 \% \\
(303 / 409)\end{array}$ & $\begin{array}{l}66.1 \% \\
(123 / 186)\end{array}$ & 0.046 & $\begin{array}{l}75.0 \% \\
(105 / 140)\end{array}$ & $\begin{array}{l}77.9 \% \\
(60 / 77)\end{array}$ & 0.629 & $\begin{array}{l}83.8 \% \\
(119 / 142)\end{array}$ & $\begin{array}{l}56.0 \% \\
(14 / 25)\end{array}$ & $\begin{array}{l}71.1 \% \\
(212 / 298)\end{array}$ & $\begin{array}{l}72.3 \% \\
(52 / 84)\end{array}$ & 0.001 \\
\hline Hymenoplasty & $\begin{array}{l}73.9 \% \\
(303 / 410)\end{array}$ & $\begin{array}{l}68.0 \% \\
(134 / 197)\end{array}$ & 0.131 & $\begin{array}{l}73.6 \% \\
(109 / 148)\end{array}$ & $\begin{array}{l}83.3 \% \\
(65 / 78)\end{array}$ & 0.100 & $\begin{array}{l}75.8 \% \\
(113 / 149)\end{array}$ & $\begin{array}{l}57.7 \% \\
(15 / 26)\end{array}$ & $\begin{array}{l}74.3 \% \\
(225 / 303)\end{array}$ & $\begin{array}{l}70.4 \% \\
(57 / 81)\end{array}$ & 0.238 \\
\hline Whitening & $\begin{array}{l}89.1 \% \\
(351 / 394)\end{array}$ & $\begin{array}{l}79.5 \% \\
(151 / 190)\end{array}$ & 0.002 & $\begin{array}{l}83.9 \% \\
(120 / 143)\end{array}$ & $\begin{array}{l}92.0 \% \\
(69 / 75)\end{array}$ & 0.095 & $\begin{array}{l}91.0 \% \\
(132 / 145)\end{array}$ & $\begin{array}{l}76.0 \% \\
(19 / 25)\end{array}$ & $\begin{array}{l}86.9 \% \\
(252 / 290)\end{array}$ & $\begin{array}{l}79.3 \% \\
(65 / 82)\end{array}$ & 0.036 \\
\hline
\end{tabular}


Table 1 Percentage of participants who answered that a specific procedure did not or rarely had a medical indication (continuation)

\begin{tabular}{|c|c|c|c|c|c|c|c|}
\hline & $\begin{array}{l}\text { Considering/had } \\
\text { plastic surgery } \\
\%(n / N)\end{array}$ & $\begin{array}{l}\text { Differentiation } \\
\%(n / N)\end{array}$ & & & & & \\
\hline & Yes & No & $p$ & Student & Resident & Specialist & $p$ \\
\hline $\begin{array}{l}\text { Vaginal laser } \\
\text { (atrophy) }\end{array}$ & $\begin{array}{l}24.6 \% \\
(43 / 175)\end{array}$ & $\begin{array}{l}22.8 \% \\
(91 / 400)\end{array}$ & 0.635 & $\begin{array}{l}15.8 \% \\
(33 / 209)\end{array}$ & $\begin{array}{l}24.6 \% \\
(44 / 179)\end{array}$ & $\begin{array}{l}28.1 \% \\
(61 / 217)\end{array}$ & 0.008 \\
\hline $\begin{array}{l}\text { Nymphoplasty } \\
\text { (reduction) }\end{array}$ & $\begin{array}{l}27.7 \% \\
(46 / 166)\end{array}$ & $\begin{array}{l}26.6 \% \\
(99 / 381)\end{array}$ & 0.674 & $\begin{array}{l}15.7 \% \\
(31 / 197)\end{array}$ & $\begin{array}{l}28.1 \% \\
(48 / 171)\end{array}$ & $\begin{array}{l}32.2 \% \\
(67 / 208)\end{array}$ & $<0.001$ \\
\hline $\begin{array}{l}\text { Reduction of the } \\
\text { labia majora }\end{array}$ & $\begin{array}{l}38,2 \% \\
(68 / 178)\end{array}$ & $\begin{array}{l}37.9 \% \\
(153 / 404)\end{array}$ & 0.940 & $\begin{array}{l}23.8 \% \\
(48 / 202)\end{array}$ & $\begin{array}{l}36.3 \% \\
(66 / 182)\end{array}$ & $\begin{array}{l}51.1 \% \\
(115 / 225)\end{array}$ & $<0.001$ \\
\hline Clitoral hood surgery & $\begin{array}{l}39.3 \% \\
(68 / 173)\end{array}$ & $\begin{array}{l}43.1 \% \\
(169 / 392)\end{array}$ & 0.398 & $\begin{array}{l}27.8 \% \\
(54 / 194)\end{array}$ & $\begin{array}{l}40.1 \% \\
(71 / 177)\end{array}$ & $\begin{array}{l}53.6 \% \\
(119 / 222)\end{array}$ & $<0.001$ \\
\hline Clitoral surgery & $\begin{array}{l}39.1 \% \\
(68 / 174)\end{array}$ & $\begin{array}{l}47.6 \% \\
(180 / 378)\end{array}$ & 0.061 & $\begin{array}{l}27.7 \% \\
(53 / 191)\end{array}$ & $\begin{array}{l}42.7 \% \\
(73 / 171)\end{array}$ & $\begin{array}{l}60.6 \% \\
(132 / 218)\end{array}$ & $<0.001$ \\
\hline Vaginal rejuvenation & $\begin{array}{l}46.3 \% \\
(82 / 177)\end{array}$ & $\begin{array}{l}49.4 \% \\
(200 / 405)\end{array}$ & 0.497 & $\begin{array}{l}45.1 \% \\
(93 / 206)\end{array}$ & $\begin{array}{l}55.0 \% \\
(99 / 180)\end{array}$ & $\begin{array}{l}44.2 \% \\
(100 / 226)\end{array}$ & 0.065 \\
\hline Laser (tightening) & $\begin{array}{l}51.4 \% \\
(89 / 173)\end{array}$ & $\begin{array}{l}54.0 \% \\
(214 / 396)\end{array}$ & 0.568 & $\begin{array}{l}45.6 \% \\
(94 / 206)\end{array}$ & $\begin{array}{l}57.6 \% \\
(102 / 177)\end{array}$ & $\begin{array}{l}54.2 \% \\
(117 / 216)\end{array}$ & 0.050 \\
\hline $\begin{array}{l}\text { Nymphoplasty } \\
\text { (augmentation) }\end{array}$ & $\begin{array}{l}61.3 \% \\
(98 / 160)\end{array}$ & $\begin{array}{l}58.9 \% \\
(219 / 372)\end{array}$ & 0.608 & $\begin{array}{l}45.0 \% \\
(85 / 189)\end{array}$ & $\begin{array}{l}63.0 \% \\
(104 / 165)\end{array}$ & $\begin{array}{l}66.8 \% \\
(137 / 205)\end{array}$ & $<0.001$ \\
\hline $\begin{array}{l}\text { Augmentation of } \\
\text { the labia majora }\end{array}$ & $\begin{array}{l}66.1 \% \\
(113 / 171)\end{array}$ & $\begin{array}{l}68.3 \% \\
(267 / 391)\end{array}$ & 0.607 & $\begin{array}{l}56.8 \% \\
(109 / 192)\end{array}$ & $\begin{array}{l}70.5 \% \\
(124 / 176)\end{array}$ & $\begin{array}{l}71.4 \% \\
(157 / 220)\end{array}$ & 0.003 \\
\hline “G-spot" augmentation & $\begin{array}{l}66.5 \% \\
(115 / 173)\end{array}$ & $\begin{array}{l}72.6 \% \\
(278 / 383)\end{array}$ & 0.143 & $\begin{array}{l}60.8 \% \\
(121 / 199)\end{array}$ & $\begin{array}{l}70.1 \% \\
(122 / 174)\end{array}$ & $\begin{array}{l}81.8 \% \\
(166 / 203)\end{array}$ & $<0.001$ \\
\hline Liposuction & $\begin{array}{l}69.5 \% \\
(123 / 177)\end{array}$ & $\begin{array}{l}73.0 \% \\
(290 / 397)\end{array}$ & 0.381 & $\begin{array}{l}62.9 \% \\
(127 / 202)\end{array}$ & $\begin{array}{l}76.1 \% \\
(134 / 176)\end{array}$ & $\begin{array}{l}76.0 \% \\
(165 / 217)\end{array}$ & 0.003 \\
\hline Hymenoplasty & $\begin{array}{l}74.2 \% \\
(132 / 178)\end{array}$ & $\begin{array}{l}72.3 \% \\
(290 / 401)\end{array}$ & 0.646 & $\begin{array}{l}64.9 \% \\
(131 / 202)\end{array}$ & $\begin{array}{l}73.7 \% \\
(132 / 179)\end{array}$ & $\begin{array}{l}77.0 \% \\
(174 / 226)\end{array}$ & 0.017 \\
\hline Whitening & $\begin{array}{l}84.9 \% \\
(146 / 172)\end{array}$ & $\begin{array}{l}88.3 \% \\
(339\end{array}$ & 0.267 & $\begin{array}{l}81.8 \% \\
(157 / 192)\end{array}$ & $\begin{array}{l}89.7 \% \\
(156 / 174)\end{array}$ & $\begin{array}{l}86.7 \% \\
(189 / 218)\end{array}$ & 0.088 \\
\hline
\end{tabular}

Abbreviation: ObGyn, Gynaecologists/Obstetricians.

Note: Includes only specialists.

[521/664]); vaginal atrophy (69.9\% [464/664]); quality of life (66.3\% [440/664]); and sexual pain (61.4\% [408/664]). There were no differences when the stratification for gender was performed (data not shown). Plastic surgeons, when compared with gynecologists, were more likely to consider the possible benefits of these procedures, and the differences were statistically significant regarding the improvements in: sexual function (92.9\% [26/28] versus 65.2\% [101/155]; $p=0.003)$; quality of life $(85.7 \%[24 / 28]$ versus $52.3 \%$ [81/155]; $p=0.001])$; sexual pain $(78.6 \%$ [22/28] versus

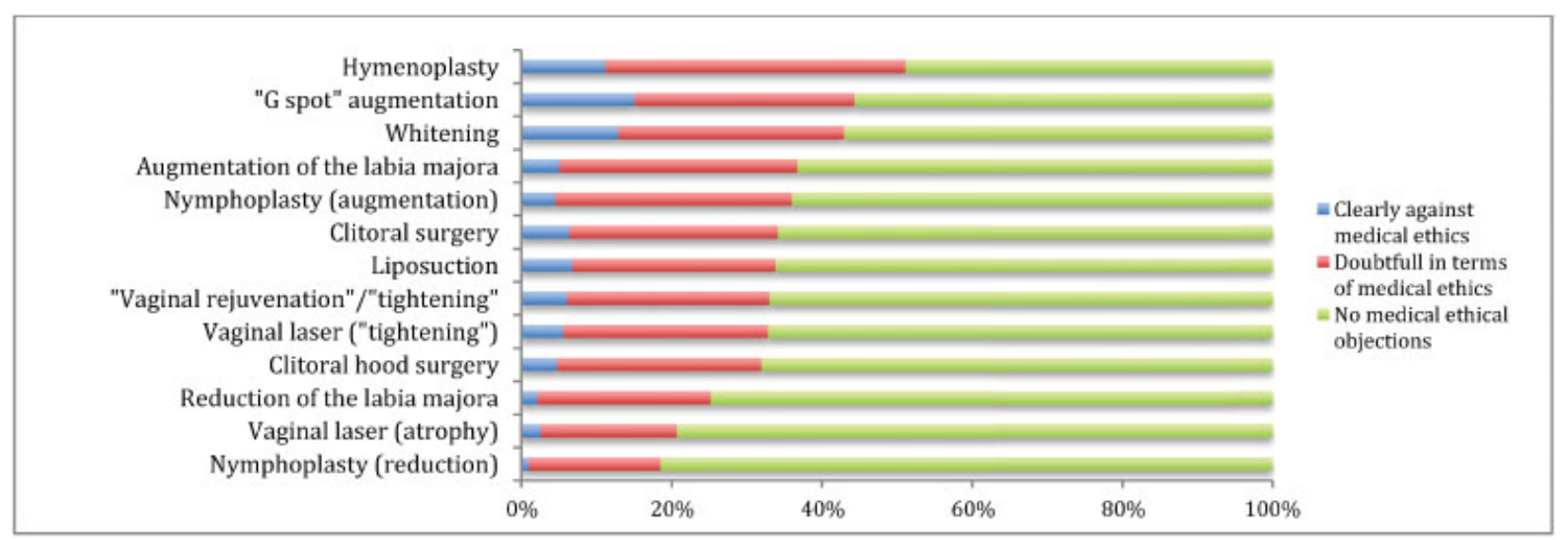

Fig. 2 Participants' opinion in terms of ethical objections for the performance of VVAPs. 
420 Survey on Aesthetic Vulvovaginal Procedures Vieira-Baptista et al.

Table 2 Percentage of participants who considered that there may be ethical issues concerning the performance of specific vulvovaginal aesthetic procedures

\begin{tabular}{|c|c|c|c|c|c|c|c|c|c|c|c|}
\hline & \multicolumn{3}{|l|}{$\begin{array}{l}\text { Gender } \\
\%(n / N)\end{array}$} & \multicolumn{3}{|c|}{$\begin{array}{l}\text { Private practice } \\
\%(n / N)\end{array}$} & \multicolumn{5}{|l|}{$\begin{array}{l}\text { Specialty } \\
\%(n / N)\end{array}$} \\
\hline & Female & Male & $p$ & Yes & No & $P$ & ObGyn & $\begin{array}{l}\text { Plastic } \\
\text { surgery }\end{array}$ & $\begin{array}{l}\text { Other } \\
\text { non-surgical }\end{array}$ & $\begin{array}{l}\text { Other } \\
\text { surgical }\end{array}$ & $p$ \\
\hline $\begin{array}{l}\text { Nymphoplasty } \\
\text { (reduction) }\end{array}$ & $\begin{array}{l}16.9 \% \\
(58 / 343)\end{array}$ & $\begin{array}{l}21.6 \% \\
(37 / 171)\end{array}$ & 0.193 & $\begin{array}{l}20.6 \% \\
(13 / 63)\end{array}$ & $\begin{array}{l}22.7 \% \\
(30 / 132)\end{array}$ & 0.742 & $\begin{array}{l}21.3 \% \\
(30 / 141)\end{array}$ & $\begin{array}{l}8.7 \% \\
(2 / 21)\end{array}$ & $\begin{array}{l}17.1 \% \\
(41 / 240)\end{array}$ & $\begin{array}{l}19.2 \% \\
(15 / 78)\end{array}$ & 0.468 \\
\hline $\begin{array}{l}\text { Vaginal laser } \\
\text { (atrophy) }\end{array}$ & $\begin{array}{l}19.9 \% \\
(73 / 366)\end{array}$ & $\begin{array}{l}22.3 \% \\
(39 / 175)\end{array}$ & 0.530 & $\begin{array}{l}26.6 \% \\
(37 / 139)\end{array}$ & $\begin{array}{l}23.1 \% \\
(15 / 65)\end{array}$ & 0.589 & $\begin{array}{l}29.6 \% \\
(40 / 135)\end{array}$ & $\begin{array}{l}8.3 \% \\
(2 / 24)\end{array}$ & $\begin{array}{l}17.0 \% \\
(45 / 265)\end{array}$ & $\begin{array}{l}22.0 \% \\
(18 / 82)\end{array}$ & 0.011 \\
\hline $\begin{array}{l}\text { Reduction of the } \\
\text { labia majora }\end{array}$ & $\begin{array}{l}25.0 \% \\
(91 / 364)\end{array}$ & $\begin{array}{l}25.7 \% \\
(47 / 183)\end{array}$ & 0.862 & $\begin{array}{l}33.8 \% \\
(48 / 142)\end{array}$ & $\begin{array}{l}29.9 \% \\
(20 / 67)\end{array}$ & 0.569 & $\begin{array}{l}41.3 \% \\
(59 / 143)\end{array}$ & $\begin{array}{l}12.5 \% \\
(3 / 24)\end{array}$ & $\begin{array}{l}19.1 \% \\
(51 / 267)\end{array}$ & $\begin{array}{l}25.0 \% \\
(20 / 80)\end{array}$ & $<0.001$ \\
\hline Clitoral hood surgery & $\begin{array}{l}33.5 \% \\
(119 / 355)\end{array}$ & $\begin{array}{l}29.0 \% \\
(53 / 183)\end{array}$ & 0.283 & $\begin{array}{l}43.3 \% \\
(61 / 141)\end{array}$ & $\begin{array}{l}39.7 \% \\
(25 / 63)\end{array}$ & 0.632 & $\begin{array}{l}48.2 \% \\
(66 / 137)\end{array}$ & $\begin{array}{l}17.4 \% \\
(4 / 23)\end{array}$ & $\begin{array}{l}26.8 \% \\
(70 / 261)\end{array}$ & $\begin{array}{l}28.9 \% \\
(24 / 83)\end{array}$ & $<0.001$ \\
\hline Laser (tightening) & $\begin{array}{l}33.9 \% \\
(121 / 357)\end{array}$ & $\begin{array}{l}30.7 \% \\
(54 / 176)\end{array}$ & 0.458 & $\begin{array}{l}40.3 \% \\
(56 / 139)\end{array}$ & $\begin{array}{l}38.7 \% \\
(24 / 62)\end{array}$ & 0.833 & $\begin{array}{l}49.3 \% \\
(66 / 134)\end{array}$ & $\begin{array}{l}12.5 \% \\
(3 / 24)\end{array}$ & $\begin{array}{l}27.6 \% \\
(71 / 257)\end{array}$ & $\begin{array}{l}31.3 \% \\
(26 / 83))\end{array}$ & $<0.001$ \\
\hline Vaginal rejuvenation & $\begin{array}{l}34.0 \% \\
(123 / 362)\end{array}$ & $\begin{array}{l}31.3 \% \\
(56 / 180)\end{array}$ & 0.504 & $\begin{array}{l}38.0 \% \\
(54 / 142)\end{array}$ & $\begin{array}{l}31.2 \% \\
(20 / 64)\end{array}$ & 0.348 & $\begin{array}{l}44.9 \% \\
(62 / 138)\end{array}$ & $\begin{array}{l}16.7 \% \\
(4 / 24)\end{array}$ & $\begin{array}{l}28.6 \% \\
(74 / 259)\end{array}$ & $\begin{array}{l}32.9 \% \\
(28 / 85)\end{array}$ & 0.003 \\
\hline Liposuction & $\begin{array}{l}34.5 \% \\
(123 / 357)\end{array}$ & $\begin{array}{l}32.6 \% \\
(56 / 172)\end{array}$ & 0.666 & $\begin{array}{l}43.8 \% \\
(57 / 130)\end{array}$ & $\begin{array}{l}44.8 \% \\
(30 / 67)\end{array}$ & 0.901 & $\begin{array}{l}54.3 \% \\
(69 / 127)\end{array}$ & $\begin{array}{l}16.7 \% \\
(4 / 24)\end{array}$ & $\begin{array}{l}29.6 \% \\
(77 / 260)\end{array}$ & $\begin{array}{l}24.1 \% \\
(20 / 83)\end{array}$ & $<0.001$ \\
\hline Clitoral surgery & $\begin{array}{l}35.0 \% \\
(122 / 349)\end{array}$ & $\begin{array}{l}32.6 \% \\
(58 / 178)\end{array}$ & 0.587 & $\begin{array}{l}45.3 \% \\
(63 / 139)\end{array}$ & $\begin{array}{l}41.9 \% \\
(26 / 62)\end{array}$ & 0.655 & $\begin{array}{l}51.5 \% \\
(70 / 136)\end{array}$ & $\begin{array}{l}13.6 \% \\
(3 / 22)\end{array}$ & $\begin{array}{l}29.5 \% \\
(75 / 254)\end{array}$ & $\begin{array}{l}28.4 \% \\
(23 / 81)\end{array}$ & $<0.001$ \\
\hline $\begin{array}{l}\text { Nymphoplasty } \\
\text { (augmentation) }\end{array}$ & $\begin{array}{l}35.1 \% \\
(119 / 339)\end{array}$ & $\begin{array}{l}37.8 \% \\
(65 / 172)\end{array}$ & 0.550 & $\begin{array}{l}50.4 \% \\
(66 / 131)\end{array}$ & $\begin{array}{l}46.0 \% \\
(29 / 63)\end{array}$ & 0.570 & $\begin{array}{l}54.7 \% \\
(76 / 139)\end{array}$ & $\begin{array}{l}18.2 \% \\
(4 / 22)\end{array}$ & $\begin{array}{l}28.7 \% \\
(69 / 240)\end{array}$ & $\begin{array}{l}36.7 \% \\
(29 / 79)\end{array}$ & $<0.001$ \\
\hline $\begin{array}{l}\text { Augmentation of } \\
\text { the labia majora }\end{array}$ & $\begin{array}{l}37.1 \% \\
(132 / 356)\end{array}$ & $\begin{array}{l}36.0 \% \\
(64 / 178)\end{array}$ & 0.800 & $\begin{array}{l}47.5 \% \\
(66 / 139)\end{array}$ & $\begin{array}{l}50.0 \% \\
(32 / 64)\end{array}$ & 0.739 & $\begin{array}{l}56.5 \% \\
(78 / 138)\end{array}$ & $\begin{array}{l}17.4 \% \\
(4 / 23)\end{array}$ & $\begin{array}{l}29.3 \% \\
(76 / 259)\end{array}$ & $\begin{array}{l}37.0 \% \\
(30 / 81)\end{array}$ & $<0.001$ \\
\hline Whitening & $\begin{array}{l}43.5 \% \\
(154 / 354)\end{array}$ & $\begin{array}{l}41.7 \% \\
(75 / 180)\end{array}$ & 0.685 & $\begin{array}{l}52.9 \% \\
(73 / 138)\end{array}$ & $\begin{array}{l}55.4 \% \\
(36 / 65)\end{array}$ & 0.740 & $\begin{array}{l}57.8 \% \\
(78 / 135)\end{array}$ & $\begin{array}{l}26.1 \% \\
(6 / 23)\end{array}$ & $\begin{array}{l}39.4 \% \\
(102 / 259)\end{array}$ & $\begin{array}{l}41.0 \% \\
(34 / 83)\end{array}$ & 0.001 \\
\hline $\begin{array}{l}\text { "G-spot" } \\
\text { augmentation }\end{array}$ & $\begin{array}{l}45.4 \% \\
(164 / 361)\end{array}$ & $\begin{array}{l}42.0 \% \\
(71 / 169)\end{array}$ & 0.460 & $\begin{array}{l}59.2 \% \\
(77 / 130)\end{array}$ & $\begin{array}{l}52.3 \% \\
(34 / 65)\end{array}$ & 0.357 & $\begin{array}{l}68.0 \% \\
(87 / 128)\end{array}$ & $\begin{array}{l}30.4 \% \\
(7 / 23)\end{array}$ & $\begin{array}{l}36.9 \% \\
(96 / 260)\end{array}$ & $\begin{array}{l}42.9 \% \\
(36 / 84)\end{array}$ & $<0.001$ \\
\hline Hymenoplasty & $\begin{array}{l}53.7 \% \\
(198 / 369)\end{array}$ & $\begin{array}{l}45.9 \% \\
(85 / 185)\end{array}$ & 0.087 & $\begin{array}{l}58.5 \% \\
(83 / 142)\end{array}$ & $\begin{array}{l}62.7 \% \\
(42 / 67)\end{array}$ & 0.560 & $\begin{array}{l}65.0 \% \\
(91 / 140)\end{array}$ & $\begin{array}{l}37.5 \% \\
(9 / 24)\end{array}$ & $\begin{array}{l}48.5 \% \\
(132 / 272)\end{array}$ & $\begin{array}{l}48.1 \% \\
(39 / 81)\end{array}$ & 0.004 \\
\hline
\end{tabular}

\begin{tabular}{|c|c|c|c|c|c|c|c|}
\hline & \multicolumn{3}{|c|}{$\begin{array}{l}\text { Considering/had plastic surgery } \\
\%(n / N)\end{array}$} & \multicolumn{4}{|c|}{$\begin{array}{l}\text { Differentiation } \\
\%(n / N)\end{array}$} \\
\hline & Yes & No & $p$ & Student & Resident & Specialist & $p$ \\
\hline Nymphoplasty (reduction) & $\begin{array}{l}16.4 \% \\
(25 / 152)\end{array}$ & $\begin{array}{l}20.5 \% \\
(69 / 336)\end{array}$ & 0.289 & $\begin{array}{l}15.1 \% \\
(26 / 172)\end{array}$ & $\begin{array}{l}17.7 \% \\
(26 / 147)\end{array}$ & $\begin{array}{l}22.1 \% \\
(43 / 195)\end{array}$ & 0.223 \\
\hline Vaginal laser (atrophy) & $\begin{array}{l}15.2 \% \\
(25 / 164)\end{array}$ & $\begin{array}{l}23.0 \% \\
(81 / 352)\end{array}$ & 0.042 & $\begin{array}{l}16.5 \% \\
(30 / 182)\end{array}$ & $\begin{array}{l}19.4 \% \\
(30 / 155)\end{array}$ & $\begin{array}{l}25.5 \% \\
(52 / 204)\end{array}$ & 0.082 \\
\hline Reduction of the labia majora & $\begin{array}{l}19.1 \% \\
(31 / 162)\end{array}$ & $\begin{array}{l}28.1 \% \\
(101 / 359)\end{array}$ & 0.029 & $\begin{array}{l}19.6 \% \\
(35 / 179)\end{array}$ & $\begin{array}{l}22.0 \% \\
(35 / 179)\end{array}$ & $\begin{array}{l}32.5 \% \\
(68 / 209)\end{array}$ & 0.007 \\
\hline Clitoral hood surgery & $\begin{array}{l}25.8 \% \\
(42 / 121) \\
\end{array}$ & $\begin{array}{l}34.4 \% \\
(120 / 329)\end{array}$ & 0.051 & $\begin{array}{l}24.6 \% \\
(44 / 179) \\
\end{array}$ & $\begin{array}{l}27.1 \% \\
(42 / 155) \\
\end{array}$ & $\begin{array}{l}42.2 \% \\
(86 / 204)\end{array}$ & $<0.001$ \\
\hline Laser (tightening) & $\begin{array}{l}27.3 \% \\
(44 / 161)\end{array}$ & $\begin{array}{l}35.7 \% \\
(124 / 347)\end{array}$ & 0.061 & $\begin{array}{l}24.7 \% \\
(45 / 182)\end{array}$ & $\begin{array}{l}33.3 \% \\
(50 / 150)\end{array}$ & $\begin{array}{l}39.8 \% \\
(80 / 201)\end{array}$ & 0.007 \\
\hline Vaginal rejuvenation & $\begin{array}{l}26.7 \% \\
(43 / 161)\end{array}$ & $\begin{array}{l}35.8 \% \\
(127 / 355)\end{array}$ & 0.042 & $\begin{array}{l}29.2 \% \\
(54 / 185)\end{array}$ & $\begin{array}{l}33.8 \% \\
(51 / 151)\end{array}$ & $\begin{array}{l}35.9 \% \\
(74 / 206)\end{array}$ & 0.359 \\
\hline Liposuction & $\begin{array}{l}25.3 \% \\
(41 / 162)\end{array}$ & $\begin{array}{l}37.1 \% \\
(130 / 350)\end{array}$ & 0.008 & $\begin{array}{l}27.5 \% \\
(50 / 182)\end{array}$ & $\begin{array}{l}28.0 \% \\
(42 / 150)\end{array}$ & $\begin{array}{l}44.2 \% \\
(87 / 197)\end{array}$ & 0.001 \\
\hline Clitoral surgery & $\begin{array}{l}25.8 \% \\
(41 / 159)\end{array}$ & $\begin{array}{l}37.7 \% \\
(129 / 342)\end{array}$ & 0.009 & $\begin{array}{l}25.7 \% \\
(45 / 175)\end{array}$ & $\begin{array}{l}30.5 \% \\
(46 / 151)\end{array}$ & $\begin{array}{l}44.3 \% \\
(89 / 201)\end{array}$ & $<0.001$ \\
\hline Nymphoplasty (augmentation) & $\begin{array}{l}30.9 \% \\
(46 / 149)\end{array}$ & $\begin{array}{l}39.0 \% \\
(131 / 336)\end{array}$ & 0.087 & $\begin{array}{l}27.1 \% \\
(46 / 170)\end{array}$ & $\begin{array}{l}29.3 \% \\
(43 / 147)\end{array}$ & $\begin{array}{l}49.0 \% \\
(95 / 194)\end{array}$ & $<0.001$ \\
\hline Augmentation of the labia majora & $\begin{array}{l}28.9 \% \\
(46 / 159)\end{array}$ & $\begin{array}{l}40.7 \% \\
(142 / 349)\end{array}$ & 0.011 & $\begin{array}{l}29.9 \% \\
(53 / 177)\end{array}$ & $\begin{array}{l}29.2 \% \\
(45 / 154)\end{array}$ & $\begin{array}{l}48.3 \% \\
(98 / 203)\end{array}$ & $<0.001$ \\
\hline Whitening & $\begin{array}{l}33.5 \% \\
(54 / 161)\end{array}$ & $\begin{array}{l}47.6 \% \\
(166 / 349)\end{array}$ & 0.003 & $\begin{array}{l}34.5 \% \\
(61 / 177)\end{array}$ & $\begin{array}{l}38.3 \% \\
(59 / 154)\end{array}$ & $\begin{array}{l}53.7 \% \\
(109 / 203)\end{array}$ & $<0.001$ \\
\hline “G-spot” augmentation & $\begin{array}{l}35.2 \% \\
(58 / 165)\end{array}$ & $\begin{array}{l}47.7 \% \\
(166 / 348)\end{array}$ & 0.007 & $\begin{array}{l}34.1 \% \\
(62 / 182)\end{array}$ & $\begin{array}{l}40.5 \% \\
(62 / 153)\end{array}$ & $\begin{array}{l}56.9 \% \\
(111 / 195)\end{array}$ & $<0.001$ \\
\hline Hymenoplasty & $\begin{array}{l}47.9 \% \\
(81 / 169)\end{array}$ & $\begin{array}{l}52,9 \% \\
(190 / 359)\end{array}$ & 0.284 & $\begin{array}{l}42.7 \% \\
(79 / 185)\end{array}$ & $\begin{array}{l}49.4 \% \\
(79 / 160)\end{array}$ & $\begin{array}{l}59.8 \% \\
(125 / 209)\end{array}$ & 0.003 \\
\hline
\end{tabular}

Abbreviation: ObGyn, Gynaecologists/Obstetricians. 


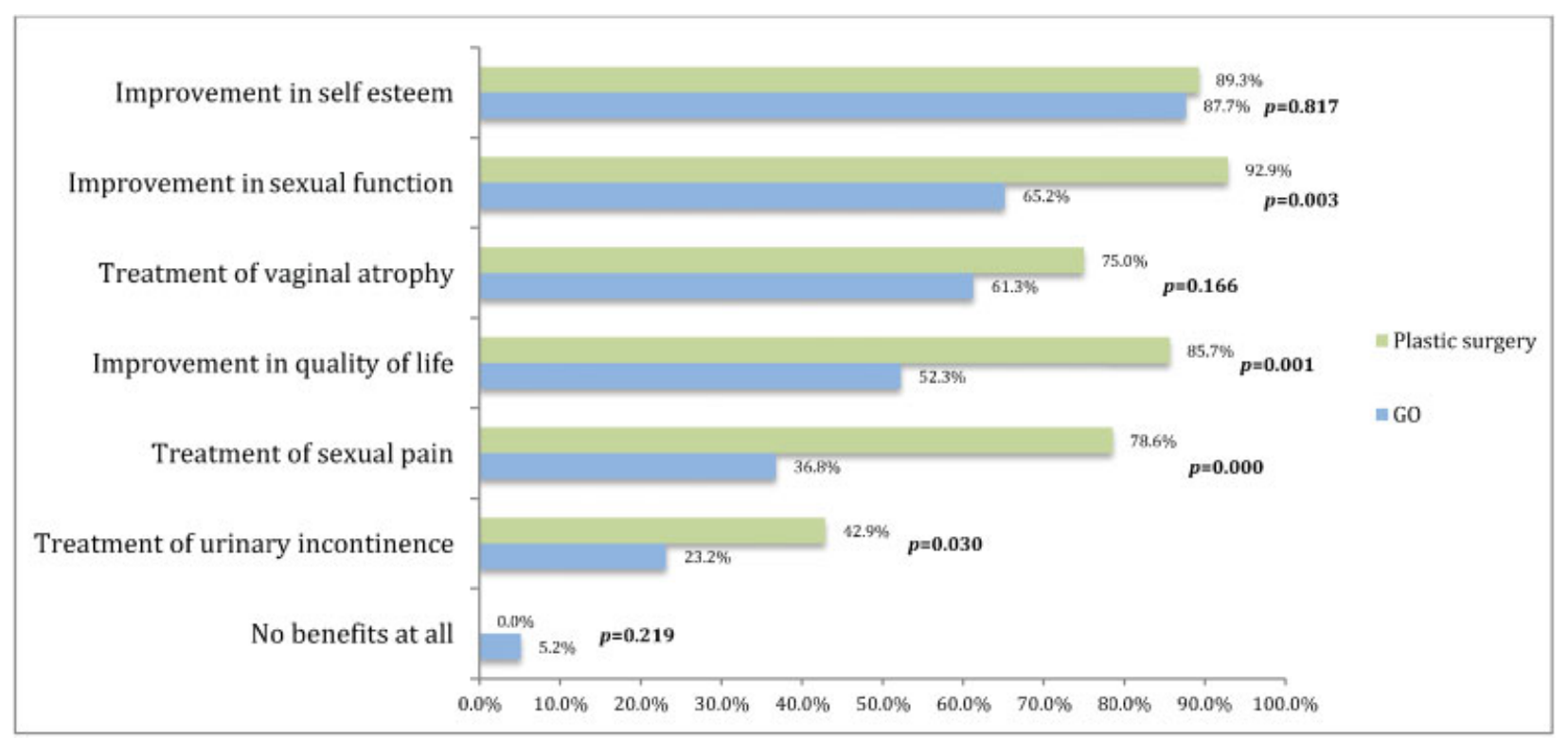

Fig. 3 Percentage of participants who considered that aesthetic procedures can have a positive impact in several questions (total includes all participants).

$36.8 \%[57 / 155], p=0.000)$; and urinary incontinence $(42.9 \%$ [12/28] versus $23.2 \%, p=0.030$ ) (-Fig. 3 ).

Most participants disagreed that VVAPs fit into the World Health Organization's (WHO) definition of female genital mutilation (51.4\% [341/664]). The majority agreed, at least partially, that: these procedures should not be performed in patients under the age of 18 years old (582/664 [87.6\%]); they should be considered in the same way as surgery at any other anatomical site (568/664 [85.5\%]); all women should be evaluated by a psychiatrist/sexologist prior to surgery (552/664 [83.1\%]); if performed, these surgeries should take place in public hospitals (381/664 [57.3\%]); advertising them should be forbidden (339/664 [51.0\%]) (-Fig. 4).

\section{Discussion}

The primary objective of this study was to evaluate the opinion of medical students and doctors on the existence of any medical justification or ethical concerns about the performance of VVAPs. The secondary outcomes included the determining factors that influenced their answers, and which potential impacts the participants considered these procedures can have.

For most VVAPs, more than half of the participants considered that there is never or there rarely is any medical justification to perform the procedures. Despite this common opinion, the percentage of participants who considered VVAPs to be unethical was much lower, and most even considered that VVAPs could have a positive impact in terms of selfesteem, sexual function, vaginal atrophy, quality of life, and sexual pain.

Vulvar whitening was considered the procedure with the least scientific background supporting its performance. It is frequently performed along with anal whitening, ${ }^{1,10}$ and is not exempt from complications. It was followed by hymenoplasty, liposuction of the mons pubis, "G-spot" augmentation,

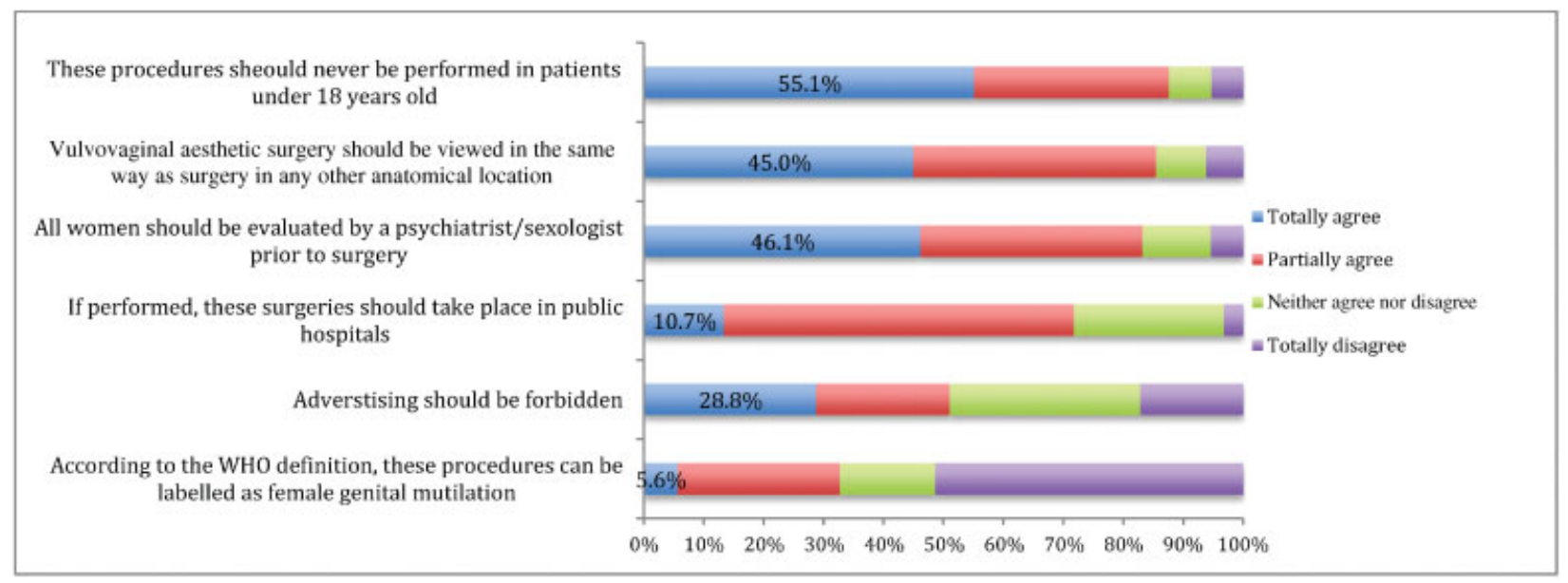

Fig. 4 Participants' agreement with several questions concerning VVAPs. 
augmentation of the labia majora, augmentation of the labia minora, and the use of laser for vaginal tightening. The existence of the "G-spot" itself is debatable, and all procedures aiming at its augmentation, despite being offered by some providers, are considered useless and unethical - just like laser vaginal tightening. 5,11,12 Hymenoplasty was suggested by Reziciner as a way to prevent recurrent post-coital cystitis, ${ }^{13}$ but without any scientific support to recommend it. Although there is never any medical indication to perform it, it has been life-saving for some Muslim girls, and this highlights how important relative ethical issues can be in different communities. ${ }^{14}$ Liposuction can be considered after significant weight loss. ${ }^{15}$ Augmentation of the labia majora and minora is often offered as an aesthetic procedure for aging women - especially with the use of fillers or autologous fat; despite the absence of studies, it can be considered in very special cases (marked loss of weight, neoplasia, hidradenitis suppurative, antiretroviral use). ${ }^{15,16}$

On the contrary, only a quarter or less of the respondents considered that there is no scientific evidence for the use of laser for the treatment of vaginal atrophy and for nymphoplasty. Despite the wide commercialization and use of laser for the treatment of vaginal atrophy, ${ }^{17}$ there have been no randomized, case control studies published. The long-term effects and safety have not been demonstrated so far. Nymphoplasty is probably the most performed VVAP, and the one with most published papers. However, there is still no definition of labial hypertrophy, ${ }^{18-20}$ no studies comparing surgery and non-intervention, as well as no data about the long-term satisfaction or late complications (scar retraction in menopause, for example).

Despite the heterogeneity of the sample, there was a good correlation between the opinion of the participants and the evidence or lack thereof in the literature. However, some factors influenced the opinion of the participants, namely the specialty and the degree of differentiation, as plastic surgeons and students were more likely to consider that there were medical indications for the procedures. On the other hand, gynecologists were more likely to raise doubts about medical indications. Gender, not having a private practice, and having had or considering having plastic surgery did not play a significant role in the opinions. The general overview changed when they were questioned if the performance of such procedures was unethical or not: more than half of the subjects only raised ethical issues about hymenoplasty. Concerning the medical justification, despite minor variations in the order, the results were fairly similar to those obtained previously. Unlike what was found in the previous question, the participants who had had or had considered having plastic surgery (nearly one third of them) were less likely to raise ethical questions about the procedures. While personal beliefs did not seem to affect the opinion in terms of the medical reason for the procedures, they had an effect when the ethics question was asked. This shows the importance and relativity of one of the four ethical principles, the principle of autonomy: ${ }^{21,22}$ the participants state that there is no medical reason for the performance of the procedures, but respect one's right to undergo them. However, the autonomy principle implies informed consent, and it cannot exist in the absence of scientific data (about complications, outcomes, etc.). It is generally assumed that the risk associated with most procedures is low (nonmaleficence principle), and that they can have some benefits (beneficence principle). However, the few studies in this area do not support the idea that the risk is low enough to overcome the non-maleficence principle. ${ }^{10,23-25}$ It must be kept in mind that the beneficence principle is secondary to the autonomy one, but the perceptions of the benefits may not be real, and there are no long-term satisfaction studies. ${ }^{22}$

Almost all participants stated that the performance of VVAPs can have advantages. Systematically, gynecologists considered VVAPs less likely to have benefits than plastic surgeons. It is relevant to state that $61 \%$ of all participants (79\% of plastic surgeons and $37 \%$ of gynecologists) considered that some of these procedures can treat sexual pain. We highlight the dangers of the performance of VVAPs without a previous evaluation by a gynecologist to exclude vulvar and/ or pelvic floor diseases. It is not uncommon to encounter in the clinical practice women with vulvodynia attributing their symptoms to the size of their labia minora. Failure to recognize lichen sclerosus can lead to unexpected results, as the disease tends to manifest in the scars (isomorphism).

More than $80 \%$ of the participants agreed that VVAPs should not be performed in patients under the age of 18 years old. Full genital growth and development is not achieved before that age, so VVAPs should never be performed in adolescents (patients with genital malformations are special cases that should be evaluated by adolescent gynecology experts). ${ }^{6,7,26}$

The claim that VVAPs are not different from procedures in other anatomical regions was also accepted by the majority of participants. Therefore, the ethical concern threshold must be raised to the same level as other procedures (breast augmentation, rhinoplasty, etc.). However, the vulva is a part of the body that is not directly exposed; therefore, many women do not know the normal range of anatomical variation. In reality, most women seeking surgery are anatomically normal. ${ }^{27}$

The need for previous evaluation by a sexologist/psychiatrist was acknowledged by nearly $75 \%$ of the participants. Body dysmorphic disorder is quite rare in the population in general, but is very common in women seeking plastic surgery. ${ }^{28}$ These patients are likely to be dissatisfied with their surgical outcome and go on to have repeated procedures. ${ }^{29}$

In Portugal, most VVAPs are performed in private settings, without insurance coverage. This clearly does not fit the justice principle, as not everyone can have access to undergo these procedures. More than half of the respondents considered that these procedures should be performed in public hospitals, where there is a finer triage of the patients and thorough psychiatric and gynecologic evaluations are performed.

Only $41 \%$ of participants agreed with the statement that advertising puts pressure in women; ${ }^{22}$ however some medical societies have already recommended against it. ${ }^{8}$

The WHO has defined female genital mutilation as "all procedures involving partial or total removal of the external female genitalia, or other injury to the female genital organs for non-medical reasons". ${ }^{30}$ Given that there is no medical reason to perform most of the discussed procedures, they can be included in this definition ${ }^{31}$-with serious legal implications in 
most countries. Although only one third of the respondents agreed with this statement, this highlights the need for clear definitions and guidelines for the procedures, for the protection of both the patients and doctors involved in them.

The weak points of the present study include the fact that there may have been several biases in the participants' enrolment: those interested in the subject and the younger ones (who are more likely to use the internet) may have been more prone to answer - as shown by the fact that the distribution of the answers was balanced between the different grades of differentiation, despite the fact that medical doctors comprise the largest group. The lack of validated scores to evaluate one's opinion/attitudes toward these procedures can make it more difficult for future comparisons.

Medical doctors and medical students acknowledge the lack of scientific support for the performance of VVAPs. However, most of them do not raise ethical objections for their performance, especially if they are less specialized, are plastic surgeons, or have themselves had or considered having plastic surgery. This, in part, may be due to an assumed potential benefit, despite the inexistence of reliable clinical data.

\section{Acknowledgments}

The authors would like to thank to Sociedade Portuguesa de Ginecologia (Portuguese Society of Gynecology), and Mr. Peter Greenhouse, FRCOG, from Bristol, UK, for his assistance with the text. The authors have received no funding for the preparation or writing of this paper.

\section{References}

1 Vieira-Baptista P, Lima-Silva J, Beires J. "Intimate surgery”: what is done and under which scientif basis? Acta Obstet Ginecol Port. 2015;9(05):393-399

2 Liao LM, Taghinejadi N, Creighton SM. An analysis of the content and clinical implications of online advertisements for female genital cosmetic surgery. BMJ Open 2012;2(06):e001908

3 Vieira-Baptista P. “Cirurgia íntima” - tempo de impor limites. Acta Obstet Ginecol Port. 2014;8(03):223-225

4 Sharp G, Tiggemann M, Mattiske J. Factors that influence the decision to undergo labiaplasty: media, relationships, and psychological well-being. Aesthet Surg J 2016;36(04):469-478

5 Committee on Gynecologic Practice, American College of Obstetricians and Gynecologists. ACOG Committee Opinion No. 378: Vaginal "rejuvenation" and cosmetic vaginal procedures. Obstet Gynecol 2007;110(03):737-738

6 Female genital cosmetic surgery: a resource for general practitioners and other health professionals. Melbourne: The Royal Australian College of General Practitioners; 2015

7 British Society for Paediatric \& Adolescent Gynaecology [Internet]. Position Statement: labial reduction surgery (Labiaplasty) on adolescents. 2013 [cited 2016 Dec 10]. Available from: http:// www.britspag.org/sites/default/files/downloads/Labiaplasty\% 20\%20final\%20Position\%20Statement.pdf

8 Society of Obstetricians and Gynaecologists of Canada. Female genital cosmetic surgery: SOGC Policy Statement, 300. J Obstet Gynaecol Can [Internet]. 2013 [cited 2016 Feb 12];35(12):e1-e5.
Available from: https://sogc.org/wp-content/uploads/2013/09/ December2013-CPG300-ENG-Online_final.pdf

9 Eysenbach G. Improving the quality of Web surveys: the Checklist for Reporting Results of Internet E-Surveys (CHERRIES). J Med Internet Res 2004;6(03):e34

10 Cihantimur B, Herold C. Genital beautification: a concept that offers more than reduction of the labia minora. Aesthetic Plast Surg 2013;37(06):1128-1133

11 Puppo V, Gruenwald I. Does the G-spot exist? A review of the current literature. Int Urogynecol J Pelvic Floor Dysfunct 2012; 23(12):1665-1669

12 Kilchevsky A, Vardi Y, Lowenstein L, Gruenwald I. Is the female G-spot truly a distinct anatomic entity? J Sex Med 2012;9(03):719-726

13 Reziciner S. [Prevention of recurrent post-coital cystitis using hymenoplasty]. Ann Urol (Paris) 1988;22(06):446-451 French

14 Ahmadi A. Ethical issues in hymenoplasty: views from Tehran's physicians. J Med Ethics 2014;40(06):429-430

15 Alter GJ. Management of the mons pubis and labia majora in the massive weight loss patient. Aesthet Surg J 2009;29(05):432-442

16 Lapalorcia LM, Podda S, Campiglio G, Cordellini M. Labia majora labioplasty in HIV-related vaginal lipodystrophy: technique description and literature review. Aesthetic Plast Surg 2013;37(04):711-714

17 Sokol ER, Karram MM. An assessment of the safety and efficacy of a fractional $\mathrm{CO} 2$ laser system for the treatment of vulvovaginal atrophy. Menopause 2016;23(10):1102-1107

18 Goodman MP. Female genital cosmetic and plastic surgery: a review. J Sex Med 2011;8(06):1813-1825

19 Ostrzenski A. Selecting aesthetic gynecologic procedures for plastic surgeons: a review of target methodology. Aesthetic Plast Surg 2013;37(02):256-265

20 Radman HM. Hypertrophy of the labia minora. Obstet Gynecol 1976;48(1, Suppl)78S-79S

21 Gillon R. Medical ethics: four principles plus attention to scope. BMJ 1994;309(6948):184-188

22 Goldstein AT, Jutrzonka SL. Ethical considerations of female genital plastic/cosmetic surgery. In: Goodman MP, editor. Female genital plastic and cosmetic surgery. Hoboken: John Wiley \& Sons; 2016. p. 39-44

23 Alter GJ. Aesthetic labia minora and clitoral hood reduction using extended central wedge resection. Plast Reconstr Surg 2008; 122(06):1780-1789

24 Rouzier R, Louis-Sylvestre C, Paniel BJ, Haddad B. Hypertrophy of labia minora: experience with 163 reductions. Am J Obstet Gynecol 2000;182(1 Pt 1):35-40

25 Goodman MP, Placik OJ, Benson RH III, et al. A large multicenter outcome study of female genital plastic surgery. J Sex Med 2010; 7(4 Pt 1):1565-1577

26 Lloyd J, Crouch NS, Minto CL, Liao LM, Creighton SM. Female genital appearance: "normality" unfolds. BJOG 2005;112(05):643-646

27 McDougall LJ. Towards a clean slit: how medicine and notions of normality are shaping female genital aesthetics. Cult Health Sex 2013;15(07):774-787

28 Tadisina KK, Chopra K, Singh DP. Body dysmorphic disorder in plastic surgery. Eplasty 2013;13:ic48

29 Likes WM, Sideri M, Haefner H, Cunningham P, Albani F. Aesthetic practice of labial reduction. J Low Genit Tract Dis 2008;12(03): 210-216

30 World Health Organization [Internet]. Eliminating female genital mutilation: an interagency statement: UNAIDS, UNDP, UNECA, UNESCO, UNFPA, UNHCHR, UNHCR, UNICEF, UNIFEM, WHO. GenevaWHO2008 [cited 2014 Aug 29]. Available from: http://apps.who. int/iris/bitstream/10665/43839/1/9789241596442_eng.pdf

31 Conroy RM. Female genital mutilation: whose problem, whose solution? BMJ 2006;333(7559):106-107 